\title{
Afectos y conductas sexuales en adolescentes: diferencias y semejanzas en dos culturas, España y Colombia*
}

\section{Sexual feelings and behaviors in adolescents: differences and similarities in two cultures, Spain and Colombia}

\author{
FÉLIX LóPEZ \\ Universidad de Salamanca, España \\ ORCID: http://orcid.org/0000-0002-8547-9043 \\ Rodrigo J. CARCEDO \\ Universidad de Salamanca, España \\ Noelia Fernández-Rouco \\ Universidad de Salamanca, España \\ María-Claudia Caballero \\ Universidad Industrial de Santander, Colombia
}

a Autor de correspondencia. Correo electrónico: flopez@usal.es

Para citar este artículo: López, F., Carcedo, R., Fernández-Rouco, N., \& Caballero, M. (2017). Afectos y conductas sexuales en adolescentes: diferencias y semejanzas en dos culturas, España y Colombia. Universitas Psychologica, 16(4), 1-11. https: //doi.org/10.11144/Javeriana.upsy16-4.acsa

\section{RESUMEN}

Este trabajo pretende identificar las posibles diferencias de género en afectos y conductas sexuales de España y Colombia. La muestra total fue de 508 participantes de centros de educación secundaria, 270 en España y 238 en Colombia, de edades entre los 15 y 18 años y con una media de edad de 16.4 años. Los resultados obtenidos muestran un doble patrón en varones y mujeres, tanto en España como en Colombia, especialmente en relación a los afectos sexuales y no tanto en las conductas sexuales.

Palabras clave

adolescencia; afectos sexuales; conductas sexuales; género; cultura.

\section{ABSTRACT}

This work aims to identify gender differences in sexual behaviors and feelings between adolescents living in Spain and Colombia. A total of 508 participants from secondary schools, 270 from Spain and 238 from Colombia, took part in this study. The age of the participants ranged from 15 and 18 years. The results showed a double standard for men and women, both in Spain and Colombia, especially in relation to sexual feelings rather than on sexual behavior.

Keywords

adolescence; sexual feelings; sexual behaviors; gender; culture.

Nuestra vida sexual y amorosa es el resultado de procesos fisiológicos de sexuación, y de procesos de socialización culturales en interacciones complejas. Por eso, podemos esperar semejanzas y diferencias en las conductas y afectos sexuales de los adolescentes que viven en diferentes culturas 
(Kottak, 2007). Sin embargo, los efectos de la socialización en culturas diferentes son esperables, si tenemos en cuenta que la sexualidad humana nos permite tomar decisiones sobre la forma de vivir la sexualidad, nuestras conductas y la forma de vivir nuestros afectos sexuales. Algunos trabajos transculturales entre adolescentes en España y en Colombia indican que el modo de relacionarse no dista significativamente entre adolescentes de ambos países, al menos en lo relativo a las redes sociales, altamente utilizadas en ambos países y especialmente destinadas a la exposición personal y las relaciones sociales, afectivas y sexuales (Almansa-Martínez, Fonseca, \& Castillo-Esparcia, 2013). Sin embargo, otros trabajos ponen de manifiesto algunas diferencias en la vivencia de los afectos y la sexualidad, especialmente en relación con el estatus afectivo y sexual y las expectativas asociadas a varones y mujeres de ambos países en personas migrantes desde Colombia a España (Posso \& Urrea, 2007).

Por otro lado, otros trabajos han resaltado la presencia de actitudes más liberales y/o abiertas con respecto a las relaciones afectivo-sexuales en España (Ayuso \& García, 2014) en comparación con Colombia (Gómez \& Torres, 2015).

A todo lo anterior se puede añadir que la educación formal podría contribuir a una formación similar y adecuada en materia de sexualidad, si se atiende a criterios científicos, profesionales y éticos (López, 2005). Sin embargo, ni en Colombia ni en España la educación en sexualidad (que pudiese contribuir a la formación sistemática de adolescentes en materia de afectos y conductas sexuales) forma parte del currículum educativo. Esto deja a los adolescentes en mayor medida a merced de la construcción de género que esté presente en su cultura. No obstante, incorporar la sexualidad en el contexto educativo implica la necesidad de retomar el conocimiento cultural y de las formas sexuales dentro de la representación de cada cultura (Epstein \& Johnson, 2000), de modo que puedan ajustarse las acciones a los contextos culturales específicos y también a los posibles elementos comunes o diferenciadores entre varones y mujeres.
A todo ello hemos de añadir que la adolescencia es la etapa evolutiva cuya tarea psicosocial principal es la construcción de la identidad (Adams \& Berzonsky, 2003), además de suponer el inicio en las relaciones afectivosexuales (López, 2005). Por lo tanto, se elige un momento crítico del desarrollo en el que los procesos de socialización culturales asociados a la construcción del género van a tener una especial importancia de cara a la construcción de la identidad del adolescente y, de manera más concreta, de sus afectos y comportamientos sexuales. En otras palabras, este estudio se centra en el estudio de la posible influencia de los procesos de socialización culturales asociados al género en la psique del adolescente y, más específicamente, en sus conductas y afectos sexuales.

En este sentido, el género se presenta como uno de los aspectos en los que mejor se reflejan los posibles efectos de la socialización cultural (Clark, 2010). Así, nos preguntamos: iexisten diferencias referidas a los afectos y las conductas sexuales en Colombia y en España?

Estudios previos con prepuberes muestran que chicos y chicas comparten algunos de los aspectos conductuales más importantes de la sexualidad, como la curiosidad y las conductas por motivación de placer (Larsson \& Svedin, 2002). Pero también se han encontrado evidencias a favor de la existencia de un doble patrón en las conductas sexuales prepuberales (Friedrich, Sandfort, Oosteveen, \& CohenKettenis, 2000; López, Del Campo, \& Guijo, 2003), que muestran más contenido afectivo en las conductas sexuales de las mujeres (Ballester $\&$ Gil, 2006).

En la pubertad y adolescencia, actualmente se pone de manifiesto que varones y mujeres adolescentes comparten opiniones liberales y sentimientos erotofílicos, opinan de forma muy similar y tienen conductas cada vez más semejantes (García-Vega, Menéndez, García, \& Rico, 2010).

No obstante, se mantienen algunos elementos de los patrones tradicionales vinculados a los dos sexos (Zimmer-Gembeck \& Helfand, 2008). En los estudios sobre adolescentes colombianos, 
se encuentra que las relaciones se inician, en más de la mitad de la población, en el marco de las relaciones románticas (González, 2004; Vargas-Trujillo, Henao, \& González, 2007). Los patrones se ponen de manifiesto en que la motivación amorosa, la necesidad de seleccionar una buena pareja, los cuidados y la fidelidad son más citadas por las mujeres (Caballero, 2010), que muestran una mayor tendencia a pensar que los hombres están muy dispuestos al sexo ocasional y no a establecer después una relación (Vargas-Trujillo, Rojas, \& Balanta, 2008). También los adolescentes varones se masturban más, recurren más a la pornografía, compran más actividad sexual y abusan o agreden con mucha más frecuencia que las adolescentes colombianas (Pacheco et al., 2007). No obstante, en Colombia también es necesario hacer más estudios intergeneracionales para describir y explicar las diferencias y semejanzas.

Teniendo todo esto en cuenta, iqué nos permite hablar de un doble patrón? Por un lado, las diferencias en numerosas conductas sexuales (Oliver \& Hyde, 1993), como la conducta de masturbación, mayor disposición al sexo ocasional o sin compromiso, y la búsqueda de la conducta coital, todas mayores en los varones, y la búsqueda de comunicación, caricias y juegos, mayor en las mujeres (Petersen \& Hyde, 2010). Por otro lado, las mujeres asocian la actividad sexual más a los afectos, la intimidad y el compromiso que los varones (Caballero, 2010; Faílde, Lameiras, \& Bimbela, 2008).

A todo lo anterior se puede añadir que la educación formal podría contribuir a una formación similar y adecuada en materia de sexualidad si se atiende a criterios científicos, profesionales y éticos (López, 2005). Sin embargo, ni en Colombia ni en España la educación en sexualidad (que pudiese contribuir a la formación sistemática de adolescentes en materia de afectos y conductas sexuales) forma parte del currículum educativo. No obstante, incorporar la sexualidad en el contexto educativo implica la necesidad de retomar el conocimiento de las culturas y las formas sexuales dentro de la representación de cada cultura (Epstein \& Johnson, 2000), de modo que puedan ajustarse las acciones a los contextos culturales específicos y también a los posibles elementos comunes o diferenciadores entre varones y mujeres. Por todo ello, nos parece tan importante estudiar las semejanzas y diferencias en el campo de la sexualidad en estas dos sociedades y culturas; la colombiana y la española, poniendo el énfasis precisamente en la perspectiva de género. En este sentido, nos planteamos la siguiente cuestión: iSon diferentes los varones y las mujeres adolescentes en sus afectos y conductas sexuales en España y en Colombia? Pero el interés central reside en: ¿Pueden ayudarnos estos datos a clarificar el debate sobre lo relativo a la especie humana y lo que aprende cada individuo? Identificar posibles patrones comunes y diferenciadores culturales y de género nos permitirá también establecer elementos comunes y también específicos de acción en torno a la sexualidad (en afectos y conductas).

\section{Método}

\section{Participantes}

La muestra ha sido seleccionada a partir de centros escolares de secundaria de dos ciudades del noroeste de España y del departamento de Santander de Colombia, equivalentes en cuanto al estatus socioeconómico, e incluyendo en la misma medida centros de estatus socioeconómico medio-alto y medio-bajo en ambos países.

La muestra consta de 508 adolescentes que han contestado a un cuestionario de autoinforme en condiciones de confidencialidad. Con respecto al sexo, nuestra muestra está compuesta por 265 chicos $(52.17 \%)$ y 243 $(47.83 \%)$ chicas, y por 270 españoles/as $(53.15 \%)$ y 238 colombianos/as (46.85\%). La media de edad es de 16.41 años $(\mathrm{DT}=0.99)$, comprendidos entre 15 y 18 años, de los cuales, 230 se encuentran en el grupo de edad "15-16 años" (45.28\%) y 278 en el grupo "17-18 años" (54.72\%). En cuanto a la historia de las relaciones de pareja, cabe destacar que los adolescentes que nunca han 
tenido pareja representan un $18.31 \%$ de la muestra total. Finalmente, 329 adolescentes (64.76\%) afirmaron haber mantenido algún tipo de contacto sexual, de los cuales, 10 señalaron haber mantenido contactos con personas del mismo sexo $(0.03 \%)$.

\section{Variables}

\section{Variables sociodemográficas}

En este estudio se tuvieron en cuenta variables sociodemográficas. Algunas fueron utilizadas para el análisis de los resultados. De esta manera, se ha tenido en cuenta el sexo, la nacionalidad (española y colombiana) y el grupo de edad (15-16 años y 17-18 años).

Motivación y afectos en las relaciones sexuales y de pareja

Para conocer el tipo de motivación en las relaciones de pareja y en las relaciones sexuales, así como para identificar los afectos sexuales, utilizamos los Cuestionarios de orientación sociosexual, versión original (Simpson \& Gangestad, 1991) y revisada (Penke \& Asendorpf, 2008). Hemos realizado una adaptación de ambas versiones de este instrumento con el objetivo de evaluar sus contenidos con respecto a la vivencia afectivo-sexual de los participantes. De este modo, evaluamos la motivación para mantener relaciones sexuales y de pareja con un rango de respuesta de Totalmente sexual (1) a Totalmente afectiva (3).

Por otro lado, en relación a los afectos sexuales, evaluamos la frecuencia de deseo sexual, con un rango de respuesta de Nunca (1) a Diario (5), el número de personas por las que se siente atracción, con cuatro opciones de respuesta que van desde Nadie (1) a Muchas personas (4), añadiendo el grado de enamoramiento, con tres opciones de respuesta, que contemplan desde Nada (1) a Totalmente (3).

\section{Conductas sexuales}

Para conocer el tipo y frecuencia de conductas sexuales que tenían los participantes, empleamos el instrumento de los Niveles de experiencia sexual (Schofield, 1975), reiteradamente utilizado en numerosas investigaciones hasta la actualidad. Se ha utilizado una adaptación de este instrumento, que consta de cinco niveles de experiencia sexual y que incluye desde besos, abrazos y caricias hasta el coito. Además, se añadió la especificidad dentro de las caricias genitales de contemplar si fueron por encima o por debajo de la ropa, y también la conducta de masturbación por no estar recogida en dicho instrumento, puesto que se centra en conductas interpersonales y no individuales. Sin embargo, la conducta de masturbación ha sido ampliamente estudiada en investigaciones sobre las diferencias sexuales en la conducta sexual (Ballester \& Gil, 2006). En todas ellas se preguntó si estas conductas habían estado presentes en los últimos seis meses, con un rango de respuesta de Nunca (1) a Diario (5) en el caso de la masturbación y de Nunca (1) a Casi Siempre (4) para el resto de conductas.

\section{Procedimiento}

Los procedimientos de obtención de datos se basaron en la aplicación de un cuestionario dentro de las aulas, en las que se crearon condiciones de confidencialidad y se garantizó el anonimato. El alumnado contestó al cuestionario de manera individual y con espacio suficiente respecto al resto del grupo para poder contestar con tranquilidad. Un representante del equipo de investigación estaba presente durante la recogida de datos, recordando la importancia al alumnado de contestar con sinceridad y de manera individual. El tiempo medio empleado para la explicación del cuestionario y la obtención de las respuestas a las preguntas incluidas en este trabajo fue de 30 minutos. 


\section{Análisis estadísticos}

En este trabajo se llevaron a cabo análisis descriptivos de medias y desviaciones típicas. Además, se realizaron una serie de ANOVAS univariantes 2 x 2 x 2 . Aunque se expondrán todas las interacciones significativas, se representarán exclusivamente aquellas que incluyan diferencias de género, puesto que el estudio del doble patrón de género es el objetivo principal de este trabajo. El análisis de las comparaciones post-hoc se realizó a través de la prueba de Bonferroni. Para la realización de este trabajo se utilizó el paquete estadístico SPSS 20.0.

\section{Resultados}

Los resultados muestran las diferencias de género tanto en motivaciones y afectos sexuales, como en conductas sexuales.

\section{Diferencias en motivaciones y afectos sexuales}

Se realizaron dos ANOVAS univariantes de tres factores (sexo, nacionalidad y grupo de edad) con el objetivo de analizar la motivación de los participantes para mantener relaciones de pareja y sexuales. A través del análisis de los efectos principales encontramos que las mujeres, en el grupo de Colombia y el grupo de 15-16 años afirmaron tener más motivaciones afectivas tanto para mantener relaciones de pareja como sexuales.

En cuanto al análisis de las interacciones con la motivación para las relaciones de pareja, aunque la interacción sexo*nacionalidad resultó significativa, encontramos que la interacción de segundo orden sexo*nacionalidad*edad también lo era (ver Tabla 1). Para comprender mejor el sentido de esta interacción, se han dispuesto los datos en dos figuras en función de la variable edad. Asimismo, se realizaron comparaciones post-hoc con el ajuste Bonferroni para estudiar las diferencias entre los grupos implicados en la interacción. En este sentido, en el grupo de 15-16 años, tan solo las mujeres españolas muestran una mayor motivación afectiva en sus relaciones de pareja que los varones españoles $\left(\mathrm{p}<0.001\right.$ eta $\left.^{2}=0.098\right)$ (ver Figura 1$)$, mientras que en el grupo de 17-18 años, las mujeres muestran una mayor motivación afectiva que los varones en ambas nacionalidades (españoles: $\mathrm{p}<0.001$, eta ${ }^{2}=0.026$; colombianos: $\mathrm{p}<0.001$, $\left.\mathrm{eta}^{2}=0.026\right)($ ver Figura 2).

\section{TABLA 1}

Diferencias en aspectos relacionados con los afectos sexuales según el sexo, la nacionalidad y la edad

\begin{tabular}{|c|c|c|c|c|c|c|c|c|c|c|}
\hline & \multicolumn{2}{|c|}{$\begin{array}{c}\text { Motivación en las } \\
\text { relaciones de }\end{array}$} & \multicolumn{2}{|c|}{$\begin{array}{c}\text { Motivación en las } \\
\text { relaciones }\end{array}$} & \multicolumn{2}{|c|}{ Deseo } & \multicolumn{2}{|c|}{ Atracción } & \multicolumn{2}{|c|}{ Enamoramiento } \\
\hline & F & $\mathrm{eta}^{2}$ & $\mathrm{~F}$ & $\mathrm{eta}^{2}$ & $\mathrm{~F}$ & eta $^{2}$ & $\mathrm{~F}$ & $\mathrm{eta}^{2}$ & $\mathrm{~F}$ & eta $^{2}$ \\
\hline Sexo & $57.67^{* * * *}$ & 0.105 & $38.86^{\text {*wok }}$ & 0.083 & $35.32^{\text {w*m }}$ & 0.066 & $33.86^{\text {**** }}$ & 0.065 & 0.01 & 0 \\
\hline Nacionalidad & $85.08 * * *$ & 0.147 & $58.05^{* * * *}$ & 0.119 & $69.06^{* * * *}$ & 0.122 & $10.63^{* * *}$ & 0.021 & $37.93^{* * * *}$ & 0.073 \\
\hline Edad & $7.75^{* *}$ & 0.015 & $30.48^{* * *}$ & 0.066 & $20.54^{* * *}$ & 0.04 & 0.18 & 0 & 2.74 & 0.006 \\
\hline $\begin{array}{l}\text { Sexo* } \\
\text { nacionalidad }\end{array}$ & $9.83 * *$ & 0.02 & $5.69 \%$ & 0.013 & 1.57 & 0.003 & 0.61 & 0.001 & 1.03 & 0.002 \\
\hline Sexo*edad & 0.55 & 0.001 & $6.77^{*}$ & 0.015 & $5.49^{*}$ & 0.011 & 0.13 & 0 & 0.52 & 0.001 \\
\hline $\begin{array}{l}\text { Nacionalidad* } \\
\text { edad }\end{array}$ & 0.66 & 0.001 & 0.28 & 0.001 & 2.96 & 0.006 & 0.31 & 0.001 & $6.15^{*}$ & 0.013 \\
\hline Sexo*nac.*edad & $10.82^{* *}$ & 0.021 & 0.71 & 0.002 & 3.66 & 0.007 & 3.63 & 0.007 & 0.04 & 0 \\
\hline$R^{2}$ & 0.26 & & 0.24. & & 0.205 & & 0.09 & & 0.08 & \\
\hline
\end{tabular}

\section{Figura 1}

Interacción del sexo y la nacionalidad para el grupo de 15-16 años con respecto a la motivación en las relaciones de pareja

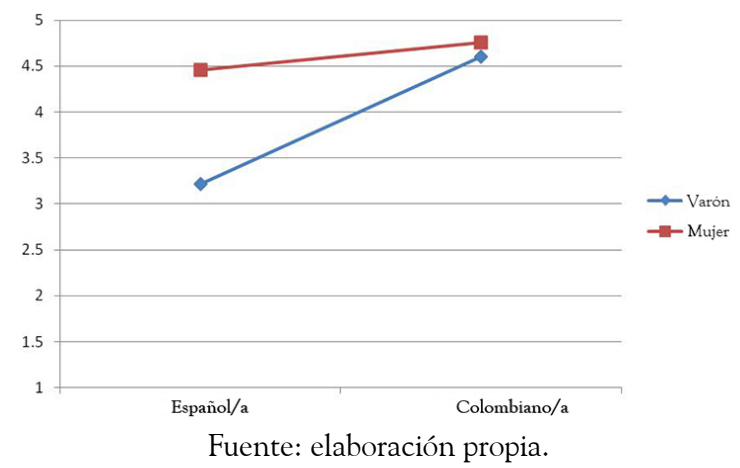

Con respecto al análisis de las interacciones con la motivación para las relaciones sexuales, encontramos que las interacciones sexo*nacionalidad y sexo*edad resultaron significativas (ver Tabla 2). Las mujeres españolas $(\mathrm{p}<0.001)$ y colombianas $(\mathrm{p}=0.012)$ (ver Figura $3)$, y las mujeres de $15-16$ y $17-18$ años $(\mathrm{p}<0.001)$ (ver Figura 4) mostraron una mayor motivación afectiva en sus relaciones sexuales que sus 
Félix López, Rodrigo J. Carcedo, Noelia Fernández-Rouco, Et al.

homólogos varones. Asimismo, se encontró que la diferencia entre las mujeres y los varones españoles $\left(\right.$ eta $\left.^{2}=0.093\right)$ era mayor que entre las mujeres y los varones colombianos $\left(\mathrm{eta}^{2}=\right.$ 0.015), y que la diferencia entre las mujeres y los varones de $15-16\left(\right.$ eta $\left.^{2}=0.074\right)$ años era también mayor que entre las mujeres y varones de 17-18 $\left(\mathrm{eta}^{2}=0.017\right)$.

\section{Figura 2}

Interacción del sexo y la nacionalidad para el grupo de 17-18 años con respecto a la motivación en las relaciones de pareja

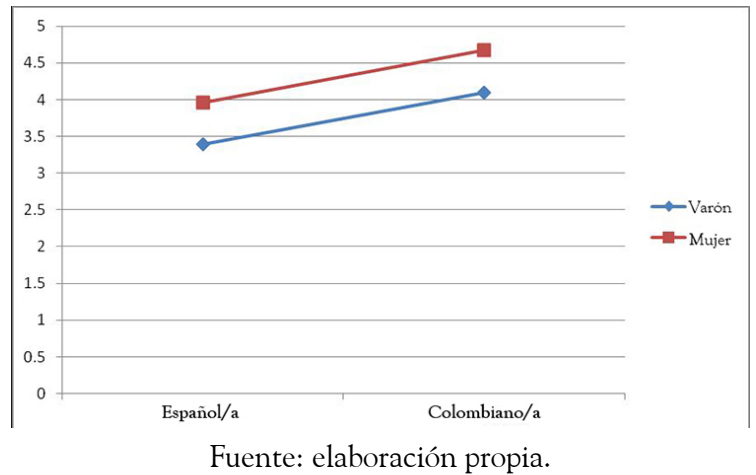

Figura 3

Interacción del sexo y la nacionalidad con respecto a la motivación en las relaciones sexuales

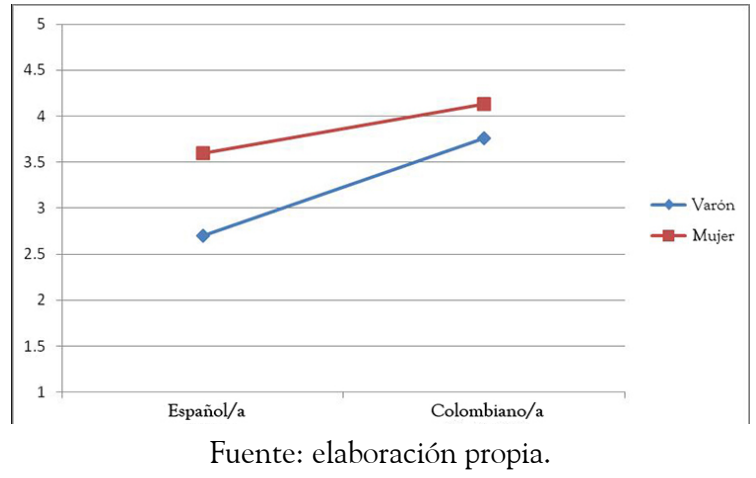

Figura 4

Interacción del sexo y edad con respecto a la motivación en las relaciones sexuales

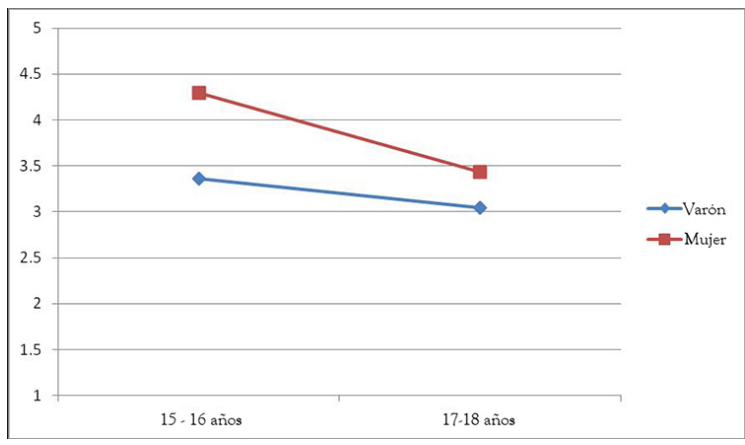

Fuente: elaboración propia.

Se realizaron tres ANOVAS univariantes de tres factores (sexo, nacionalidad y grupo de edad) con el objetivo de analizar los afectos sexuales de deseo, atracción y enamoramiento. El análisis de los efectos principales de los afectos sexuales deseo, atracción y enamoramiento mostró que las mujeres afirmaron una menor frecuencia de deseo y un menor número de personas por las que sentían atracción. Asimismo, el grupo total de los españoles afirmaron sentir deseo con mayor frecuencia, sentirse atraídos por un mayor número de personas y mostraron niveles menores de enamoramiento en comparación con el grupo total de los colombianos (ver Tabla 1).

En el caso del deseo, se encontró que la interacción sexo*edad resultó significativa (ver Tabla 1). Las mujeres de $15-16$ ( $\mathrm{p}<0.001)$ y $17-18$ años $(p=0.008)$ mostraron una menor frecuencia de deseo que sus análogos varones, si bien la diferencia entre las mujeres y varones de 15-16 años $\left(\right.$ eta $\left.^{2}=0.06\right)$ es mayor que la diferencia entre las mujeres y los varones de 17-18 años $\left(\mathrm{eta}^{2}=0.014\right)($ ver Figura 5). 
Figura 5

Interacción del sexo y la edad con respecto al deseo

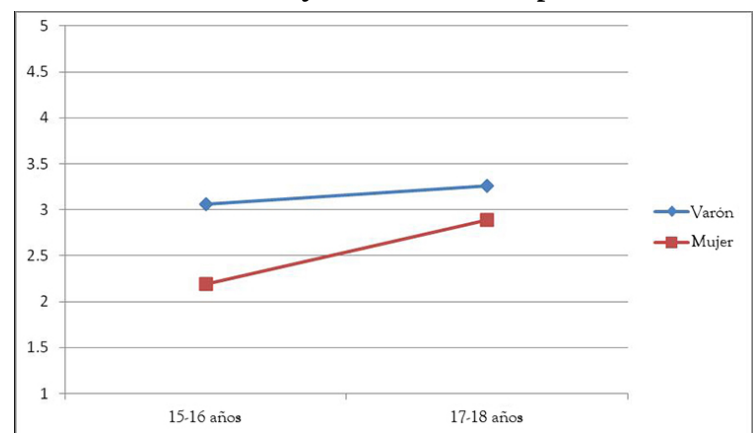

Fuente: elaboración propia.

Que estas diferencias del deseo disminuyan con la edad implica que hay factores de socialización indudables en este patrón. Por otro lado, la interacción nacionalidad*edad resultó significativa en el caso del enamoramiento (ver Tabla 1). Aunque esta interacción no sea representada en una figura por no estar relacionada con los objetivos de nuestro estudio, es importante señalar que el grupo de colombianos afirmó encontrarse enamorado en mayor grado que el grupo de los españoles, en donde esta diferencia fue mayor en el grupo de edad de $15-16$ años $\left(\mathrm{p}<0.001\right.$ eta $\left.^{2}=0.067\right)$ que en el de $17-18\left(\mathrm{p}=0.007\right.$, eta $\left.^{2}=0.015\right)$.

\section{Diferencias en conductas sexuales}

Se realizaron 7 ANOVAS univariantes de tres factores (sexo, nacionalidad y grupo de edad) con el objetivo de analizar 6 conductas sexuales interpersonales (besos, abrazos y caricias no genitales, contactos genitales por encima y por debajo de la ropa, sexo oral y coito vaginal y anal) y una conducta sexual individual (la masturbación).

Del análisis de los efectos principales se desprende que con respecto a las conductas sexuales interpersonales más frecuentes (todas las anteriores excepto el coito anal), no existen diferencias entre varones y mujeres, mientras que los españoles y el grupo de 17-18 años muestran una mayor frecuencia de estas conductas. Por otro lado, los varones y los españoles afirman una mayor frecuencia de coito anal. Finalmente, el grupo de varones y el grupo de españoles también afirma haber practicado la masturbación con mayor frecuencia (ver Tabla 2).

TABLA 2

Diferencias en conductas sexuales según el sexo, la nacionalidad y la edad

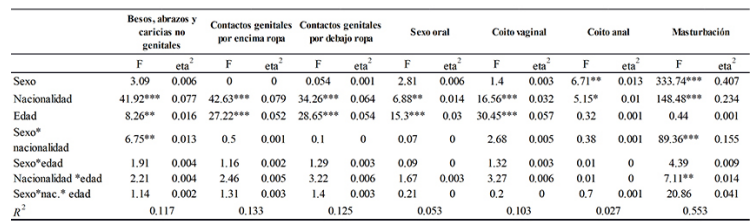

Fuente: elaboración propia.

En relación al análisis de las interacciones con los besos, abrazos y caricias no genitales, encontramos que la interacción sexo*nacionalidad resultó significativa (ver Tabla 2). Las mujeres españolas $(p=0.002)$ mostraron una mayor frecuencia de besos, abrazos y caricias que sus varones (ver Figura 6). Del mismo modo, se encontró que la diferencia entre las mujeres y los varones españoles $\left(\right.$ eta $\left.^{2}=0.02\right)$ era mayor que entre las mujeres y los varones colombianos $\left(\mathrm{eta}^{2}=0.001\right)$.

\section{Figura 6}

Interacción del sexo y la nacionalidad con respecto a los besos, abrazos y caricias no genitales

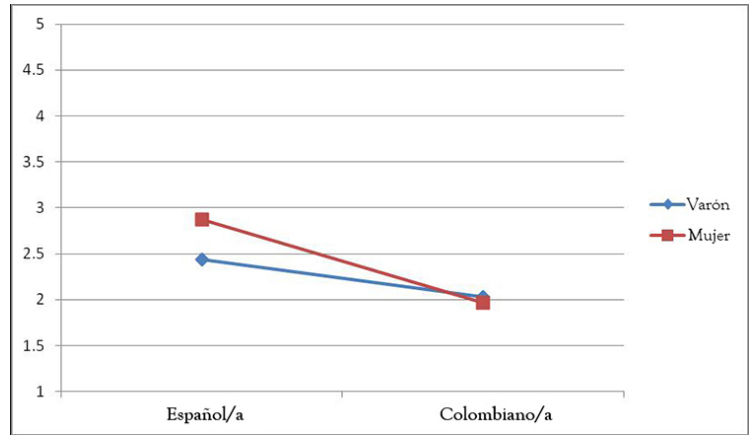

Fuente: elaboración propia.

Finalmente, en el caso de la conducta de masturbación, las interacciones sexo*nacionalidad y nacionalidad*edad resultaron significativas (ver Tabla 3). Respecto a la primera interacción, las mujeres españolas $(\mathrm{p}<0.001)$ y colombianas $(\mathrm{p}<0.001)$ mostraron una menor frecuencia de masturbación que sus 
homólogos varones (ver Figura 7). Además, se encontró que la diferencia entre las mujeres y los varones españoles $\left(\right.$ eta $\left.^{2}=0.451\right)$ era mayor que entre las mujeres y los varones colombianos $\left(\right.$ eta $\left.^{2}=0.071\right)$.

\section{Figura 7}

Interacción del sexo y la nacionalidad con respecto a la masturbación

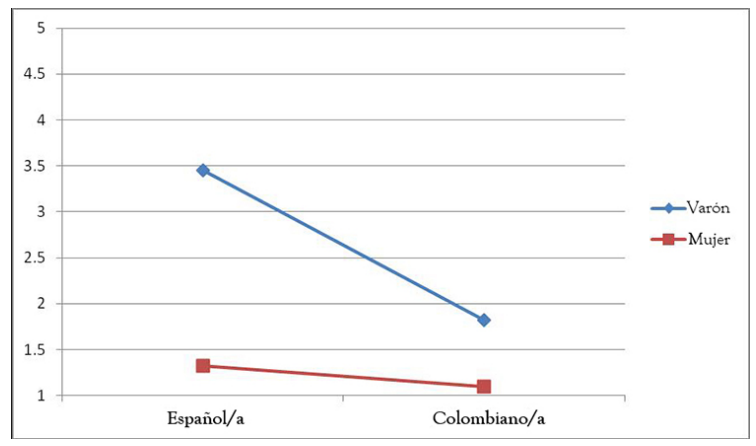

Fuente: elaboración propia.

Sin embargo, aunque la interacción nacionalidad*edad no ha sido representada en una figura, refleja que el grupo de españoles afirmó una mayor frecuencia de masturbación que el grupo de los colombianos, en donde esta diferencia fue mayor en el grupo de edad de 15-16 años $\left(\mathrm{p}<0.001\right.$ eta $\left.^{2}=0.171\right)$ que en el de 17-18 $\left(\mathrm{p}<0.001\right.$, eta $\left.^{2}=0.093\right)$.

Por último, a pesar de la influencia de las interacciones en los resultados, es importante resaltar que los efectos principales encontrados explican un mayor porcentaje de varianza de la variable dependiente que los efectos de las interacciones. En este sentido, parece que se muestran diferencias significativas entre varones y mujeres en la mayoría de los afectos y motivaciones sexuales, en donde dicho efecto no se encuentra en las conductas, salvo en el caso del coito anal y la masturbación.

\section{Discusión}

Como esperábamos de acuerdo con los trabajos realizados por numerosos autores (Burke, 2010; Clark, 2010; Kottak, 2007), los resultados apoyan algunos hechos ya encontrados anteriormente.
El primer hecho sobresaliente es que, como ya habíamos encontrado en un estudio previo con muestra española (López, Carcedo, FernándezRouco, \& Kilani, 2011), hay claras diferencias entre los hombres y las mujeres sobre la importancia concedida a los afectos y el compromiso de pareja, siempre mayor en el caso de las mujeres. Este patrón transcultural es un patrón flexible, sujeto a influencias culturales como demuestran otros dos resultados de nuestro estudio: los afectos tienen aún más importancia en las mujeres colombianas que en las españolas, y más importancia en las mujeres durante la primera adolescencia en ambos países. Este conjunto de datos nos lleva a interpretar que la base de este patrón no está únicamente en pre programaciones, sino en tradiciones ancestrales transmitidas por la cultura (más tradicional la colombiana, en este sentido, que la española) y por la familia (que tiene un mayor poder de socialización en la primera adolescencia). Todo parece indicar que las mujeres, a medida que avanzan en edad, se muestran más independientes de estos valores anclados en la tradición cultural que mantienen viva numerosas familias.

Un segundo hecho sobresaliente se refiere a las formas de vivir los afectos sexuales, deseo, atracción y enamoramiento (López, 2004). Los resultados de nuestro estudio muestran que las mujeres de ambos países afirman tener menos deseos explícitos de actividad sexual, se masturban menos y sienten atracción por un menor número de hombres, siempre en comparación con los hombres de ambos países. Nuevamente, estamos ante un patrón transcultural regulado por el sexo, pero también en este caso se hace presente el patrón tradicional en Colombia más que en España y con diferencias menores entre ambos sexos a medida que avanzan en edad, datos que corroboran nuestra interpretación anterior. Los datos sobre el enamoramiento, con mayor peso en la muestra colombiana y en la primera adolescencia van en la misma dirección, por lo que podemos decir que los afectos sexuales parecen estar regulados por un doble patrón, con diferencias entre hombres y 
mujeres, pero también por influencias culturales y de socialización familiar.

Los resultados referidos a las conductas reflejan menos diferencias entre hombres y mujeres (López et al., 2011) y menos diferencias transculturales, por lo que todo parece indicar que los factores asociados a la globalización han provocado dos efectos generales: el acceso más temprano a las conductas sexuales, incluso coitales, y un patrón de conductas cada vez más semejante entre los hombres y las mujeres, especialmente a medida que avanzan en la adolescencia.

¿Por qué esta aparente contradicción entre afectos y conductas? No tenemos una respuesta definitiva, pero pudiera ocurrir que estando en un periodo de grandes cambios en relación con la sexualidad, la regulación globalizadora transcultural de las conductas sexuales sea más efectiva por afectar más al comportamiento manifiesto que los cambios referidos a los sentimientos y a los afectos, bien porque estos estén más anclados en diferencias de sexo, bien porque dependan de factores educativos infantiles y familiares arraigados. Esto nos coloca en el centro del debate sobre este tema y nos anima a seguir haciendo investigaciones en esta misma dirección.

En este sentido, algunas de las propuestas de investigación futuras que planteamos irían en la línea de poder investigar qué ocurre a lo largo del desarrollo con la vivencia de los afectos y conductas sexuales, para desarrollar investigaciones longitudinales que permitan analizar estas posibles diferencias de género con la misma muestra a lo largo del tiempo. Por otro lado, sería interesante diseñar estrategias de intervención en sexualidad, con acento en los afectos y conductas, con el fin de poder evaluar el efecto de las intervenciones en la posible vivencia diferenciada de la sexualidad en varones y mujeres. Finalmente, resultaría especialmente interesante el diseño de acciones específicas que incidiesen en los elementos comunes entre ambas culturas, de modo que pudiesen generalizarse en ambos países.

Por otro lado, creemos que los resultados obtenidos tienen implicaciones para el bienestar de chicos y chicas en ambas culturas, en tanto que conocer cómo viven sus afectos y cómo se comportan en relación con la sexualidad varones y mujeres en España y Colombia posibilitará un mejor ajuste de las actuaciones educativas y psicosociales que podrían ponerse en marcha. En este sentido, identificar similitudes y diferencias de género y transculturales ayuda a diseñar acciones específicas eficaces y a conectar dichas acciones con la experiencia y vivencia adolescente, tanto en el ámbito educativo como en el socio-sanitario.

A la vista de los resultados de este trabajo, los programas educativos en sexualidad podrían trabajar la importancia concedida a los afectos $y$ al compromiso en las relaciones afectivosexuales, las consecuencias de las diferencias entre varones y mujeres y el modo en que se podrían abordar estas diferencias de manera ética. Este es un aspecto importante a trabajar en ambos países y, según los resultados, especialmente en Colombia.

Así mismo, también podrían trabajarse en estos programas las diferencias encontradas en los deseos de actividad sexual, frecuencia de masturbación y atracción hacia una o varias personas. En este sentido, sería importante trabajar para que, independientemente de las diferencias, chicos y chicas se sientan libres para vivir su sexualidad como así lo deseen, siempre dentro de un comportamiento ético y saludable.

De otro modo, los resultados de este trabajo permiten diseñar estrategias políticas que promuevan la implementación institucional del trabajo en sexualidad entendido de forma holística y global.

Finalmente, este trabajo permite, por un lado, estimular la propia reflexión entre los adolescentes, puesto que destinan un tiempo a pensar sobre cómo son sus afectos y conductas en los asuntos tratados y, por otro lado, permite promover el empoderamiento por hacerles partícipes de su aprendizaje en este asunto.

No podemos olvidar que el presente trabajo también ha presentado algunas dificultades que tienen que ver con la muestra, o con la propia idiosincrasia de los estudios transculturales. De este modo, no ha sido posible controlar la 
homogeneidad de la muestra entre ambos países, si bien se han tenido en cuenta criterios de elegibilidad que han tratado de minimizar los posibles efectos diferenciadores. Por otro lado, el diseño del instrumento ha de tener en cuenta las características culturales y poblacionales, aunque hemos tratado de resolverlo con el trabajo para el diseño del instrumento de investigadores en ambos países.

El presente trabajo nos lleva a preguntarnos: ¿serían en el futuro los resultados transculturales más homogéneos?, iserá el efecto globalizador tan importante como para dar paso a compartir similitudes en países como España y Colombia, no solo en conductas, sino también en afectos?

\section{Agradecimientos}

Los autores quieren expresar su agradecimiento a todos los centros educativos que voluntariamente accedieron a participar en este estudio.

\section{Referencias}

Adams, G.R., \& Berzonsky, M.D. (2003). Blackwell Handbook of Adolescence. Oxford: Blackwell Sci.

Almansa-Martínez, A., Fonseca, O., \& CastilloEsparcia, A. (2013). Redes sociales y jóvenes. Uso de Facebook en la juventud colombiana y española. Comunicar, 40 (20), 127-135. https://doi.org/10.3916/C40-2013 $-03-03$

Ayuso, L., \& García, L. (2014). Los españoles y la sexualidad en el siglo XXI. Madrid: CIS.

Ballester, R., \& Gil, M.D. (2006). La sexualidad en niños de 9 a 14 años. Psicothema, 18(1), 25-30. Recuperado de http://www.psicothe ma.com/pdf/3171.pdf

Burke, P. (2010). Hibridismo cultural. Madrid: Akal.

Caballero, M. C. (2010). Conocimientos, Actitudes y Practicas Relacionadas con la presencia de infecciones de transmisión sexual y el VIH/ SIDA en los estudiantes que han participado en el Programa de Atención y Prevención de las ITS, especialmente, VIH/SIDA (Informe de investigación no publicado). PRASI de Bienestar Universitario, Colombia.

Clark, A. (2010). Una historia de la sexualidad en Europa. Madrid: Cátedra.

Epstein, D., \& Johnson, R. (2000). Sexualidades e institución escolar. Madrid: Morata.

Faílde, J.M., Lameiras, M., \& Bimbela, J.L. (2008). Prácticas sexuales de chicos y chicas españoles de 14-24 años de edad. Gaceta Sanitaria, 22(6), 511-518.

Friedrich, W. N., Sandfort, T., Oostveen, J., \& Cohen-Kettenis, P. (2000). Cultural differences in sexual behavior: 2-6 year old Dutch and American children. Journal of Psychology and Human Sexuality, 12(1-2), 117-29.

García-Vega, E., Menéndez, E, García, P., \& Rico, R. (2010). Influencia del sexo y del género en el comportamiento sexual de una población adolescente. Psicothema, 22(4), 606-612.

Gómez, Y.G., \& Torres, V.L. (2015). Significados acerca de la sexualidad en estudiantes de psicología en Colombia. Sexualidad. Salud y Sociedad-Revista Latinoamericana, 21, 136-53.

González, G. (2004). Conocimientos, Actitudes y Prácticas en Salud Sexual y Reproductiva en Jóvenes Entre 14 Y 25 Años De Edad De Estratos 1 Y 2 del sisben de un municipio del Departamento de Cundinamarca. Acta Colombiana de Psicología, 12, 59-68.

Kottak, C. (2007). Espejo para la humanidad. Introducción a la antropología cultural. Madrid: Siglo XXI.

Larsson, I., \& Svedin, C. (2002). Teachers' and parents' report on 3 to 6 years old children's sexual behavior: a comparison. Child Abuse and Neglect, 26(3), 247-66.

López, F. (2005). La educación sexual. Madrid: Biblioteca Nueva.

López, F. (2004). Conducta sexual de mujeres y varones. Iguales y diferentes. En E. Barbera e I. Martínez (Eds.), Psicología y género (pp. 145-170). Madrid: Pearson. 
López, F., Del Campo, A., \& Guijo, V. (2003) Prepuberal sexuality. European Journal of Medical Sexology, 12, 49-65.

López, F., Carcedo, R., Fernández, N., Blazquez, M.I., \& Kilani, A. (2011). Diferencias sexuales en la sexualidad adolescente: afectos y conductas. Anales de Psicología, 27(3), 791-799.

Pacheco, C. I, Rincón, L. J., Guevara, E. E., Latorre, C., Enríquez, C., \& Nieto, J. M. (2007) Significaciones de la sexualidad y salud reproductiva en adolescentes de Bogotá. Salud Publica de México, 49(1), 45-51.

Penke, L., \& Asendorpf, J. B. (2008). Beyond global sociosexual orientations: A more differentiated look at sociosexuality and its effects on courtship and romantic relationships. Journal of Personality and Social Psychology, 95(5), 1113-1135.

Oliver, M. J.,\& Hyde, J. S. (1993). Gender differences in sexuality: A meta-analysis. Psychological Bulletin, 114(1), 29-51.

Petersen, J. L., \& Hyde, J. S. (2010). A meta-analytic review of research on gender differences in sexuality, 1993-2007. Psychological Bulletin, 136(1), 21-38.

Posso, J., \& Urrea, F. (2007). La migración internacional y los cambios en las relaciones de género y estructuras de los hogares: la migración colombiana hacia España. Papers de Sociología, 85, 109-133.

Schofield, M. (1975). El comportamiento sexual de los jóvenes. Barcelona: Fontanella.

Simpson, J. A., \& Gangestad, S. W. (1991). Individual differences in sociosexuality: Evidence for convergent and discriminant validity. Journal of Personality and Social Psychology, 60(6), 870-883.

Vargas-Trujillo, E., Henao, J., \& González, C. (2007). Toma de decisiones sexuales y reproductivas en la adolescencia. Estudio cualitativo. Acta Colombiana de Psicología, 10(1), 49-63.

Vargas-Trujillo, E., Rojas, A., \& Balanta, P. (2008). Televisión y género: Un análisis desde la perspectiva de los niños y las niñas, Mediaciones, 8, 25-39.
Zimmer-Gembeck, M. J,. \& Helfand, M. (2008). Ten years of longitudinal research on U.S. adolescent sexual behavior: The evidence for multiple pathways to sexual intercourse, and the importance of age, gender and ethnic background. Developmental Review, 28(2), 153-224.

\section{Notas}

* Artículo de investigación. 